\title{
Self-isolation among discharged emergency department patients with suspected COVID-19
}

\author{
Lulu Yang ${ }^{1} \mathbb{D} \cdot$ Devon Mitchell ${ }^{1} \cdot$ Frances Clayton $^{1} \cdot$ Paul Clerc $^{1} \cdot$ Tori Spangehl $^{1} \cdot$ Rebecca Oxland $^{2} \cdot$ Kerry Spearing $^{1}$. \\ Skye Barbic ${ }^{3,4,5}$. Gary Andolfatto ${ }^{1}$. Eric Grafstein ${ }^{1} \cdot$ Frank Scheuermeyer $^{1,2,5}$ • Andrew Kestler ${ }^{1,2,4,5}$
}

Received: 9 August 2021 / Accepted: 23 August 2021 / Published online: 5 October 2021

( ) The Author(s), under exclusive licence to Canadian Association of Emergency Physicians (CAEP)/ Association Canadienne de Médecine d'Urgence (ACMU) 2021

Keywords COVID-19 $\cdot$ Self-isolation $\cdot$ Adherence $\cdot$ Pandemic

\section{Dear Editor,}

The COVID-19 pandemic has dealt a significant blow to the health and economic well-being of the global community. Pandemic control relies heavily on self-isolation for those with confirmed or suspected illness. Modelling in Australia showed that $70 \%$ adherence to isolation in positive cases reduces and delays the epidemic peak [1]. However, studies in other counties have shown that adherence to self-isolation is suboptimal $[2,3]$.

This survey study aimed to assess self-isolation adherence in discharged emergency department (ED) patients with confirmed or suspected COVID-19, surveying individuals discharged from three EDs in Vancouver, British Columbia (BC) from May to June of 2020. We excluded individuals who were (a) institutional residents, (b) without stable housing, (c) unable to communicate in English,

Lulu Yang

lulu.yang@alumni.ubc.ca

1 Department of Emergency Medicine, University of British Columbia, Diamond Health Care Center 11th floor, 2775

Laurel Street, Vancouver, BC V5Z 1M9, Canada

2 St. Paul's Hospital Emergency Department, 1081 Burrard St, Vancouver, BC V6Z 1Y6, Canada

3 Occupational Science and Occupational Therapy, University of British Columbia, T325 - 2211 Wesbrook Mall, Vancouver, BC V6T 2A1, Canada

4 Providence Health Care Research Institute, 10th Floor - 1190 Hornby St, Vancouver, BC V6Z 2K5, Canada

5 Center for Health Evaluation and Outcome Sciences, St. Paul's Hospital, 588 - 1081 Burrard Street, Vancouver, BC V6Z 1Y6, Canada (d) non-residents of $\mathrm{BC}$, and (e) undergoing asymptomatic COVID-19 screening.

Automated reports identified 342 patients with confirmed or suspected COVID-19 during the study period. We reached 138 individuals who met criteria and offered them survey participation, 65 (47\%) of which completed the survey.

Participants were $52 \%$ female, $75 \%$ under the age of 60 , and $21 \%$ healthcare workers; $8 \%$ of participants tested positive for COVID-19. Half (56\%) reported adherence to selfisolation guidelines. The top reasons given for non-adherence were access to food, medication, and fresh air. The majority (65\%) shared their home with others, $69 \%$ of which were unable to avoid shared areas, with the challenge of limited space most often cited as the reason for non-adherence. Participants were asked seven questions to test their understanding of self-isolation rules, scoring a mean of $80 \%$.

Self-isolation is a public health requirement for those who test positive or are under investigation for COVID-19. Our results demonstrate that despite fair understanding of self-isolation rules, adherence for symptomatic individuals is partial, with almost half unable to adhere to self-isolation guidelines. This agrees with other studies conducted in the UK and Israel on self-isolation of symptomatic individuals, which show adherences of $25 \%$ and $57 \%$ respectively $[2,3]$. In agreement with previously studied responses to quarantine measures, our results demonstrate lack of access to essential supplies can lead to decreased adherence [4].

Our results raise concerns about the ability and willingness of symptomatic ED patients to adhere to self-isolation guidelines. Current public health efforts and future pandemic planning should focus on sustainable methods to support those in self-isolation with basic necessities and emphasize messaging to reduce in-home transmission and to raise overall adherence to self-isolation. 
Funding There has been no significant financial support for this work.

Data availability All data are fully available upon request.

Code availability Not applicable.

\section{Declarations}

Conflict of interest There are no conflicts of interests associated with this work.

Ethics approval This study was approved by the Research Ethics Board at the University of British Columbia, certificate number H20-01456.

Consent to participant Verbal informed consent was obtained from all participants, and electronic consent forms were documented and are available upon request.

Consent to publication All authors consent to the publication of this manuscript.

\section{References}

1. Chang SL, Harding N, Zachreson C, Cliff OM, Prokopenko M. Modelling transmission and control of the COVID-19 pandemic in Australia. Nat Commun. 2020;11:5710. https://doi.org/10.1038/ s41467-020-19393-6.

2. Smith LE, Amlôt R, Lambert H, Oliver I, Robin C, Yardley L, et al. Factors associated with adherence to self-isolation and lockdown measures in the UK: a cross-sectional survey. Public Health. 2020;187:41-52. https://doi.org/10.1016/j.puhe.2020.07.024.

3. Bodas M, Peleg K. Self-isolation compliance in the COVID-19 era influenced by compensation: findings from a recent survey in israel. Health Aff. 2020;39(6):936-41. https://doi.org/10.1377/ hlthaff.2020.00382.

4. Brooks SK, Webster RK, Smith LE, Woodland L, Wessely S, Greenberg N, et al. The psychological impact of quarantine and how to reduce it: rapid review of the evidence. Lancet. 2020;395:912-20. https://doi.org/10.1016/S0140-6736(20) 30460-8. 